\title{
MiR-486-5p Act as a Biomarker in Endometrial Carcinoma: Promotes Cell Proliferation, Migration, Invasion by Targeting MARK I
}

This article was published in the following Dove Press journal: OncoTargets and Therapy

\author{
Xiaojiao Zheng (D) \\ Kejun Xu \\ Linyan Zhu \\ Meiya Mao \\ Fubin Zhang \\ Lining Cui
}

Department of Obstetrics and Gynecology, Ningbo First Hospital, Ningbo, Zhejiang 315035, People's Republic of China
Correspondence: Lining Cui

Department of Obstetrics and

Gynecology, Ningbo First Hospital, 59

Liuting Street, Haishu District, Ningbo,

Zhejiang 315035, People's Republic of

China

Email 1935148739@qq.com
Background: miRNA expression acts as a potential biomarker in many diseases including endometrial carcinoma (EC). miR-486-5p dysregulation is observed in several tumor types, but the roles of miR-486-5p in EC are hardly ever studied.

Objective: This study aimed to analyze the expression profile of miR-486-5p in tumor tissues and serum samples of patients with EC and explore the target prediction, function analysis and validation in immortal cell lines.

Patients and Methods: A total of 42 freshly paired EC tissues, the corresponding adjacent non-neoplastic tissues and serum samples were also collected from patients with EC, and 42 matched normal serum samples were included as control group. The level of miR-486-5p expression was tested by quantitative real-time polymerase chain reaction (qRT-PCR). Cell proliferation was determined by colony formation assay and CCK-8 assay. Furthermore, functional evaluation of miR-486-5p on migration was performed by wound-healing assay and invasion was estimated by transwell invasion assay. qRT-PCR, luciferase reporter assay and Western blotting (WB) were performed to verify the targeting of MARK1 by miR-486-5p.

Results: miR-486-5p was significantly up-regulated in EC tissues and serum samples, promoting the proliferation, migration and invasive activities of EC cells by targeting MARK1.

Conclusion: These data indicated miR-486-5p as a novel molecular biomarker for diagnosing and treating EC, and MARK1 might act as a critical and functional target of miR-486-5p with the implications on cell proliferation, migration and invasiveness of EC tumor cells.

Keywords: endometrial carcinoma, miR-486-5p, MARK1, proliferation, migration, invasion

\section{Introduction}

Endometrial carcinoma (EC) is one of the most common gynecological malignancies in women, with more than 80000 newly diagnosed patients in Europe yearly. ${ }^{1}$ EC is dichotomized into two types based on estrogen levels, clinical and histopathological behavior as less aggressive and estrogen-dependent type I, highly aggressive and estrogen-independent type II. $^{2}$ Hysterectomy and bilateral salpingo-oophorectomy, chemotherapy and radiation are considered as effective treatment strategies for EC; however, it demonstrated an extremely poor prognosis. So, novel screening methods for pre-cancerous and early disease are warranted. The molecular changes that underlie EC initiation and progression are poorly understood, and so it is imperative to find key signaling pathways for novel diagnostic, prognostic and treatment strategies. ${ }^{3}$

MicroRNAs (miRNAs) are a class of short 18-25-nucleotide noncoding RNAs that post-transcriptionally regulate gene expression and are considered important in many 
fundamental biological functions. ${ }^{4,5}$ Microenvironment that favors replication and growth of miRNAs expression is regarded as an important factor. This is because they bind to partially complementary sequences that target the 3 'untranslated region ( $3^{\prime}-\mathrm{UTR}$ ) of mRNA and modulate gene expression, resulting in either mRNA degradation or translation repression or both, which therefore influences the biological function. ${ }^{5,6}$ miRNAs are new biomarkers in the pathogenesis and progression of different types of cancers. ${ }^{7,8}$ Increasing evidence has demonstrated the important link between miRNAs and cancer, in which miRNAs act as oncogene or anti-oncogene virtually in all tumors including gynecological cancers. So, these may become specific therapeutic targets in the future. 8,9

Evidence has revealed that miRNA-486-5p functions both as an oncogene and tumor suppressor in multiple tumor types. It is downregulated in non-small cell lung cancer, ${ }^{10}$ esophageal squamous cell carcinoma, ${ }^{11}$ breast cancer, ${ }^{12,13}$ gastric adenocarcinoma $^{14}$ and colorectal carcinogenesis. ${ }^{15}$ However, miR-486-5p was upregulated in cervical cancer. ${ }^{16}$ Also, other researches indicated reduced miR-486-5p expression in renal cell carcinoma $^{17,18}$ and chronic myeloid leukemia. ${ }^{19}$ Disordered expression of miR-486-5p was associated with the occurrence and development of various cancers, functions including proliferation, migration and invasion. To date, the clinical significance and the exact mechanism of miR-486-5p of EC are not fully elucidated.

We herein analyzed the expression of miR-486-5p in EC clinical samples and cell lines, investigated the roles of miR$486-5 \mathrm{p}$ in the regulation of EC cell proliferation, migration and invasion, and explored the underlying mechanisms regulated by miR-486-5p. Also, the potential roles of miR-486$5 \mathrm{p}$ in EC were investigated. More importantly, MARK1 that acts as a specific key regulator of microtubule dynamics was confirmed as a functional target of miR-486-5p.

\section{Patients and Methods}

\section{Patients and Specimen}

Forty-two matched specimens of Endometrial carcinoma and adjacent normal tissues were collected from patients enrolled at Ningbo First Hospital from 2013 to 2018. Endometrial carcinoma patients who had undergone surgery written informed consent. Endometrial carcinoma serum samples were obtained from Endometrial carcinoma patients who had provided the tissues, 42 control normal serum samples were also collected from healthy donors who came from a checkup. Forty-two samples of Endometrial carcinoma were obtained from patients who were without chemotherapy or radiotherapy before surgery, in addition, subgrouped into stage (I $\sim \mathrm{IV})$ group according to the Federation International FIGO criteria. Tissue samples after surgical removal and serum samples after centrifugal collect were both frozen immediately in liquid nitrogen and stored at $-80^{\circ} \mathrm{C}$. Clinical parameters including age, grade, FIGO stage, estrogen receptor (ER) status, and lymph node metastasis. The data are illustrated in Table 1.

Healthy donors provided written informed consent, in accordance with the Declaration of Helsinki, all tissue and serum samples were collected and all procedures were performed in accordance with the China Ethical Review Committee and Ningbo First Hospital Ethical Committee (Petition Number: 2018-R061).

\section{Cell Culture}

The endometrial carcinoma cell lines ECC-1 and HEC1-B were purchased from Boster Company (Boster, Wuhan, China). Human endometrial carcinoma cell lines AN3CA and Ishikawa were obtained from Shanghai Fuxiang Biotechnology Company (Fuxiang, Shanghai, China). Cells were cultured under condition in a humidified incubator at $37^{\circ} \mathrm{C}$ containing $5 \% \quad \mathrm{CO}_{2}$ in RPMI1640 (ECC-1, Ishikawa), DMEM (AN3CA) and

Table I Relationship Between Expression of miR-486-5p and Basic Clinical Characteristics in Tumor Tissues from Endometrial Carcinoma Patients

\begin{tabular}{|c|l|l|l|l|}
\hline \multirow{2}{*}{ Variables } & $\mathbf{n}$ & \multicolumn{2}{|l|}{$\begin{array}{l}\text { miR-486-5p } \\
\text { Expression }\end{array}$} & \multirow{2}{*}{ p value } \\
\cline { 2 - 4 } & & Low & High & \\
\hline $\begin{array}{c}\text { Age } \\
<50\end{array}$ & 25 & 16 & 9 & \multirow{2}{*}{0.735} \\
$\geq 50$ & 17 & 10 & 7 & \\
\hline $\begin{array}{l}\text { FIGO stage } \\
\text { Stage I } \\
\text { Stage II/III/IV }\end{array}$ & 34 & 10 & 24 & 0.656 \\
\hline $\begin{array}{l}\text { Grade } \\
\text { I } \\
\text { II/III }\end{array}$ & 8 & 3 & 5 & \\
\hline $\begin{array}{l}\text { Lymph nodes metastasis } \\
\text { Negative } \\
\text { Positive }\end{array}$ & 24 & 13 & 11 & 0.179 \\
\hline $\begin{array}{l}\text { ER status } \\
\text { Negative } \\
\text { Positive }\end{array}$ & 36 & 24 & 12 & \\
\hline
\end{tabular}


MEM (HEC-1-B) supplemented with 10\% fetal bovine serum (FBS)(Gibco, Auckland, NZ).

\section{Cell Transfection}

Ishikawa cells were transfected with miR-486-5p mimics or negative control and HEC-1-B were transfected with miR-486-5p inhibitor or negative control (GenePharma, Shanghai, China) for 24 or $48 \mathrm{~h}$ using Lipofectamine ${ }^{\mathrm{TM}}$ 2000 (Invitrogen, Carlsbad, USA) in accordance with the manufacturer's instructions. Primers used in the study are shown in Table 2.

\section{Cell Proliferative Capacity Assay}

Cell Counting Kit-8 (CCK-8) assay: Following transfection, cells were seeded into 96 -well culture plates $\left(2 \times 10^{3}\right.$ cells/ well). At different time points, $10 \mu \mathrm{L}$ CCK- 8 solution was added to each well and then incubated at $37^{\circ} \mathrm{C}$ for another 1-2 h (Dojindo, Kumamoto, Japan). The absorbance at 450 nm (A450) was detected using a microplate reader.

For the colony formation assay, following transfection, cells were seeded at 500 cells/well in six-well culture plates. Cells were washed thrice with phosphate-buffered saline (PBS), fixed with paraformaldehyde (4\%) for $30 \mathrm{~min}$, and then stained with crystal violet $(0.1 \%)$ for $20 \mathrm{~min}$ after $10-14$ days at $37^{\circ} \mathrm{C}$ following inoculation. Any colony with more than 50 cells was counted under a microscope. The rate of colony formation was calculated according to method: (number of colonies/number of cells inoculated) $\times 100 \%$.

\section{Cell Cycle Analysis}

For the different phases of cell-cycle analysis, transfected cells were plated in a six-well transwell plates $\left(5 \times 10^{4}\right.$ cells/ well). Cell-cycle progression was determined using a Cell Cycle Detection Kit (MultiSciences, China). After transfection for $48 \mathrm{~h}$, the cells were washed with PBS and collected by centrifugation, and then treated with $10 \mu \mathrm{L}$ permeabilization solution and $1 \mathrm{~mL}$ DNA-staining solution. Finally, incubated for $30 \mathrm{~min}$ in the dark at room temperature and analyzed via flow cytometry (BD Biosciences, USA).

\section{Wound-Healing Assay}

A wound scratch assay was used to determine the migratory ability, following transfection, cells were seeded in 6-well plates and reached confluence. Cell monolayers were scratched using sterile 200 micropipette tips. The cells were rinsed three times with PBS and afterward incubated in a $0.1 \%$ FBS medium. Images of the wound scratches were captured at each time at 0,24 and $48 \mathrm{~h}$ hours. Wound closure was calculated via measuring areas migrated by cells.

\section{Cell Invasion Assay}

Following transfection, the serum-free cells $\left(8 \times 10^{4}\right.$ cells) in serum-free DMEM medium were plated into a 24-well transwell plate into Matrigel-coated. Matrigel (BD Biosciences, Franklin Lakes, USA) was diluted (1:10) with standard medium. $500 \mu \mathrm{L}$ Standard medium supplemented with $10 \%$ FBS was placed in the lower chambers. Over a time period of $48 \mathrm{~h}$, the chamber was fixed with formalin for $30 \mathrm{~min}$ and stained with crystal violet (Beyotime Institute Biotech, Shanghai, China) for $30 \mathrm{~min}$ at room temperature, then photographed under a microscope at $200 \times$ magnification and calculated the number of penetrating cells (Leica, Wetzlar, Germany).

\section{RNA Extraction and Quantitative Real-Time PCR}

Total RNA from cultured cells, frozen serum and tissue specimens were isolated using TRizol reagent (Invitrogen, Carlsbad, USA) according to the

Table 2 Primers Used in the Study

\begin{tabular}{|l|l|l|}
\hline Identifier & Sense Primer Sequences & Antisense Primer Sequences \\
\hline miR-486-5p mimics & 5'-UCCUGACUGAGCUGCCCCGAGCGGG & \\
miR-486-5p mimics & GCAGCUCAGUACAGGAUU-3' & \\
(negative control) & 5'-UUCUCCGAACGUGUCACGUTT -3' & 5'-ACGUGACACGUUCGGAGAATT -3' \\
miR-486-5p inhibitor & 5'-CUCGGGGCAGCUCAGUACAGGA -3' \\
miR-486-5p inhibitor & 5'-CAGUACUUUUGUGUAGUACAA -3' & \\
$\begin{array}{l}\text { MARKI control) } \\
\text { Gapdh }\end{array}$ & 5'-GCTCACCACAACCTCTGCTTCC -3' & \\
\hline
\end{tabular}


manufacturer's instructions. The quality of the RNA was measured using Nanodrop. qRT-PCR assays were used to detect mRNA expression using SYBR Green Kit (TaKaRa, Dalian, China) and miRNA expression using SYBR Green miRNA Kit (GenePharma, Shanghai, China) according to the manufacturer's instructions. The U6 small nuclear RNA and gapdh mRNA were used as the references for quantification. qRT-PCR amplification was performed by using an ABI StepOne real-time PCR system. The primers used in the study for amplifying are shown in Table 2 .

\section{Luciferase Reporter Assay}

To certify directly binding of miR-486-5p to the 3'untranslated region (UTR), the wildtype (WT) or mutant reporter plasmid vector and up or up negative control were co-transfected in Ishikawa cells using Lipofectamine ${ }^{\mathrm{TM}}$ 2000 (Invitrogen, Carlsbad, USA) in 24-well plates. After transfection $48 \mathrm{~h}$, the cells were lysed. Subsequently, luciferase activity was measured using a Dual Luciferase Reporter Assay Kit (Promega, USA) according to the manufacturer's instructions. Data were normalized to the intensities of Renilla luminescence, and the results were presented as relative to luciferase activity.

\section{Western Blotting}

The cells were harvested and lysed in RIPA buffer (Solarbio, USA) containing protease inhibitors. The concentration of protein samples was tested using the bicinchoninic acid protein kit (beyotime, Shanghai, China) $0.10 \%$ sodium dodecyl sulfate-polyacrylamide gel electrophoresis (SDSPAGE) was employed to separate equal amounts of the soluble proteins, then transferred to polyvinylidene difluoride membranes (Millipore, Billerica, MA, USA) and blocked in $5 \%$ non-fat milk at room temperature for $1 \mathrm{~h}$. Membranes were incubated with rabbit monoclonal anti-MARK1 antibodies (1:1000 diluted) from Cell Signaling Technology (Beverly, MA, USA) and rabbit polyclonal anti-actin antibodies (1:1000 diluted) from Santa Cruz Biotechnology (Santa Cruz, CA, USA) overnight at $4^{\circ} \mathrm{C}$, subsequently 1 $\mathrm{h}$ incubation with appropriate goat anti-rabbit IgG-H\&L secondary antibodies (1:2000 diluted) from Abcam (Cambridge, MA, USA). The protein bands were visualized using the enhanced chemiluminescence (ECL) detection solution.

\section{Statistical Analysis}

Statistical analysis of all data was analyzed by software Statistical Package for Social Sciences (SPSS) version 22.0 (SPSS Inc., Chicago, IL, USA), performed by software GraphPad Prism version 7.0 (GraphPad, San Diego, USA). Student's $t$-test was calculated to compare when two groups, and one-way analysis of variance (ANOVA) to compare three or more groups, and clinical data were assessed using chi-square test. A P-value of less than 0.05 was considered as significantly difference.

\section{Results}

There are clearly higher levels of miR-486-5p were observed in tissue samples of EC when compared with control, as well as serum samples. As shown in Figure 1 (A), miR-486-5p was significantly overexpressed EC tissues than in matched adjacent tissues $(* * * \mathrm{P}<0.001)$. As shown in Figure 1(B), the expression of miR-486-5p was significantly increased in serum samples of EC patients when compared with controls $(* * * \mathrm{P}<0.001)$. Figure $1(\mathrm{C})$ shows a close correlation of miR-486-5p expression in between the patients' serum and tissues $(* * * \mathrm{P}<0.001)$. Figure 1(D) reveals significant results of receiver operating characteristic (ROC) analysis, indicating that the expression of serum miR-486-5p assay in EC patients was significant when compared to healthy donors $(* * * \mathrm{P}<0.001)$.

The relationship between miR-486-5p expression level and age, grade, FIGO stage, estrogen receptor (ER) status, and lymph node metastasis was analyzed in EC. Nevertheless, no association was shown between the basic clinical characteristics and the expression level of miR-486-5p in tumor tissues (Table 1).

To determine the relative expression of miR-486-5p in four EC cell lines, quantitative RT-PCR analysis was performed via small RNA fractions. As shown in Figure 2A, the relative expression level of miR-486-5p remained the highest in HEC-1-B cells and lowest in Ishikawa cells $(* * * \mathrm{P}<0.001)$. Next, miR-486-5p transfection efficiency was determined after transfection for $24 \mathrm{~h}$, and the cells were analyzed by flow cytometry. Figure $2 \mathrm{~B}$ shows overexpression of miR486-5p in Ishikawa cells and low expression of miR-486-5p in HEC-1-B cells. These data suggested that $96.97 \%$ of Ishikawa cells carried FAM-labeled miR-486-5p, and 95.98\% of HEC-1-B cells carried FAM-labeled miR-486$5 p$. Furthermore, the levels of miR-486-5p were determined by qRT-PCR (Figure 2C). The levels of mRNA expression of 
A

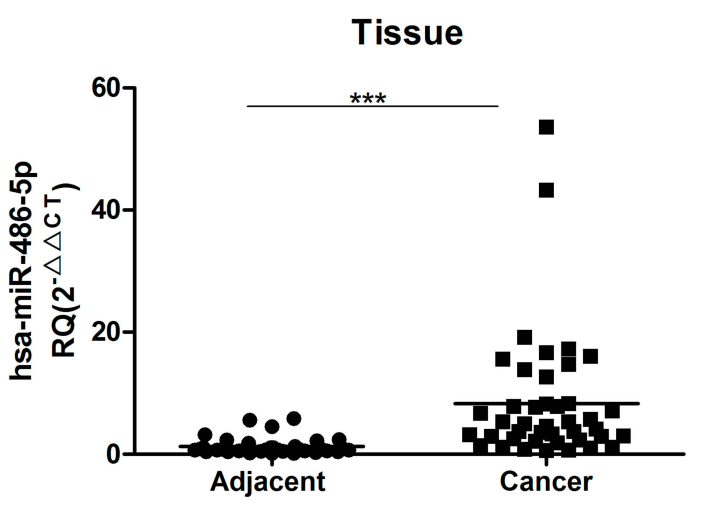

C

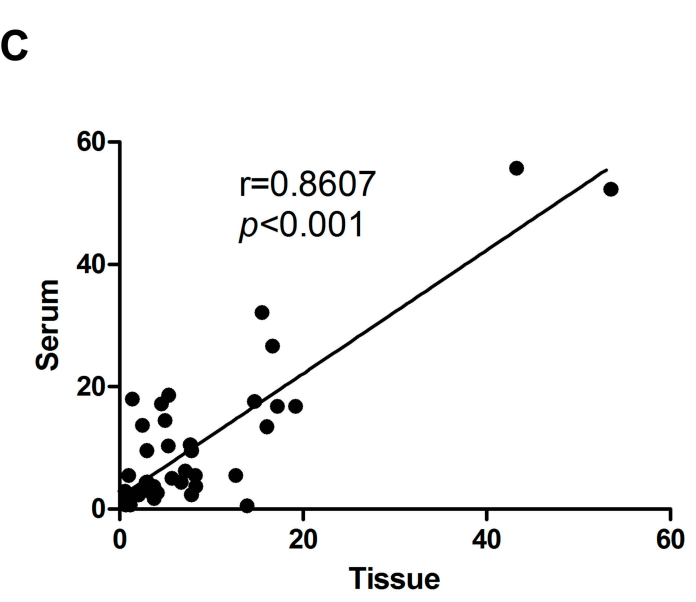

B

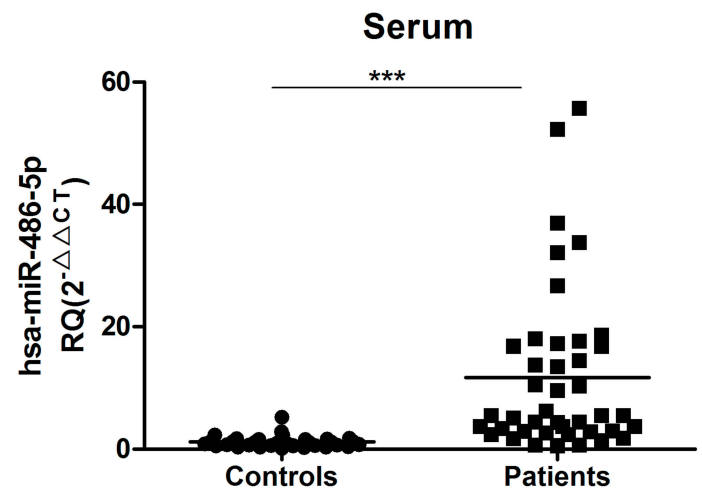

D

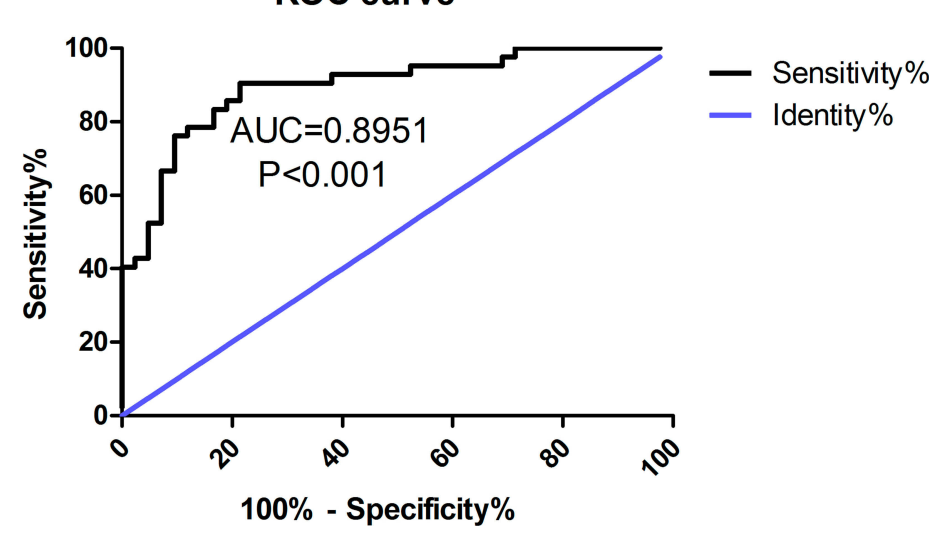

Figure I MiR-486-5p is overexpressed in serum and tumor tissues from Endometrial carcinoma patients. (A) The expression differences of miR-486-5p between endometrial carcinoma tissues and matched tumor-adjacent tissues. $* * * \mathrm{P}<0.00 \mathrm{I}$. (B) miR-486-5p expression in serum from healthy donors (Controls) and endometrial cancer patients (Patients). ${ }^{* * *} \mathrm{P}<0.00 \mathrm{I}$. (C) The correlation of miR-486-5p expression in endometrial carcinoma patients' serum and tissues. (D) ROC analysis of the serum miR-486-5p expression for endometrial carcinoma patients vs healthy subjects. Data are expressed as the mean \pm SD.

miR-486-5p were significantly increased in miR-486-5ptransfected Ishikawa cells when compared to those with mock-transfected cells $(* * * \mathrm{P}<0.001)$. The mRNA expression levels of miR-486-5p were significantly decreased in miR-486-5p-transfected HEC-1-B cells when compared with mock-transfected cells ( $* * \mathrm{P}<0.05)$.

To determine the possible role of miR-486-5p in tumor cellular proliferation, cell viability was examined through CCK-8 assay. Figure 3(A and B) demonstrated that Ishikawa cells transfected with miR-486-5p mimic had enhanced proliferation at 48,72 and $96 \mathrm{~h}$ when compared to the control groups $(* * * \mathrm{P}<0.001)$, and HEC-1-B cells transfected with miR-486-5p inhibitors inhibited the cell growth at 48,72 and $96 \mathrm{~h}$ when compared to the control groups $(* * \mathrm{P}<0.01, * * * \mathrm{P}<0.001)$. The colony formation assay was performed to determine the possible role of miR-486-5p in tumor cell proliferation. As shown in
Figure 3(C and D), cell viability was detected through colony formation assay. Overexpression of miR-486-5p has significantly promoted the clonogenicity of Ishikawa cells $(* \mathrm{P}<0.05)$. The downregulated expression of miR486-5p has significantly reduced the colony formation rate of HEC-1B cells $(* \mathrm{P}<0.05)$.

The abnormal expression of miR-486-5p has changed the G1 phase of cell cycle in tumor cells. Previous studies have shown that proliferation control is primarily achieved in the G1 phase of the cell cycle in cancer cells. ${ }^{20}$ Figure $4(\mathrm{~A}-\mathrm{C})$. Ishikawa cells transfected with miR-486-5p mimics showed a marked increase in $S$ phase cells, and so the G1 phase cells were decreased $\left({ }^{*} \mathrm{P}<0.05\right)$. Figure 4(D-F). HEC-1-B cells transfected with miR-486-5p inhibitors after $48 \mathrm{~h}$ resulted in significant increase of G1 phase cells, and so the $\mathrm{S}$ phase cells were decreased $(* \mathrm{P}<0.05)$. 
A

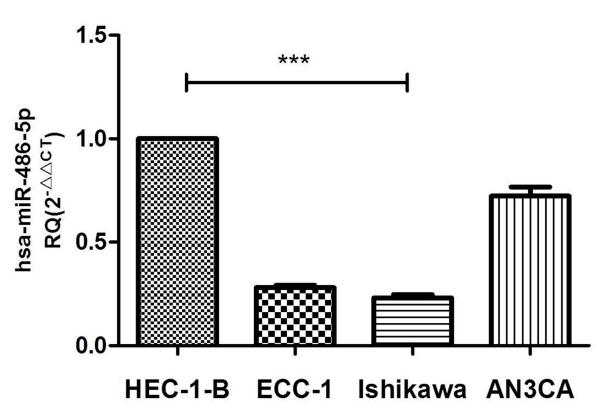

C

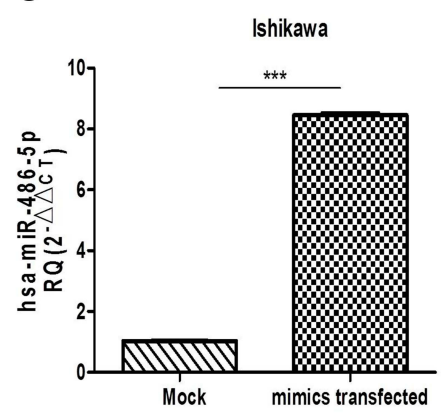

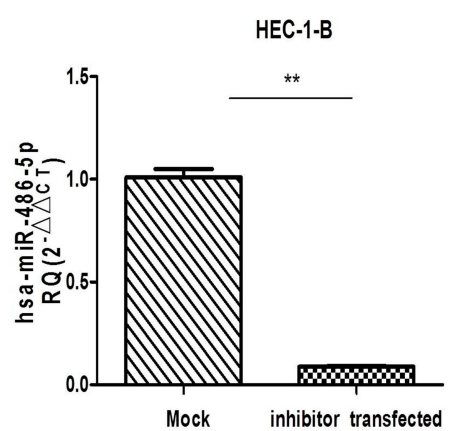

B
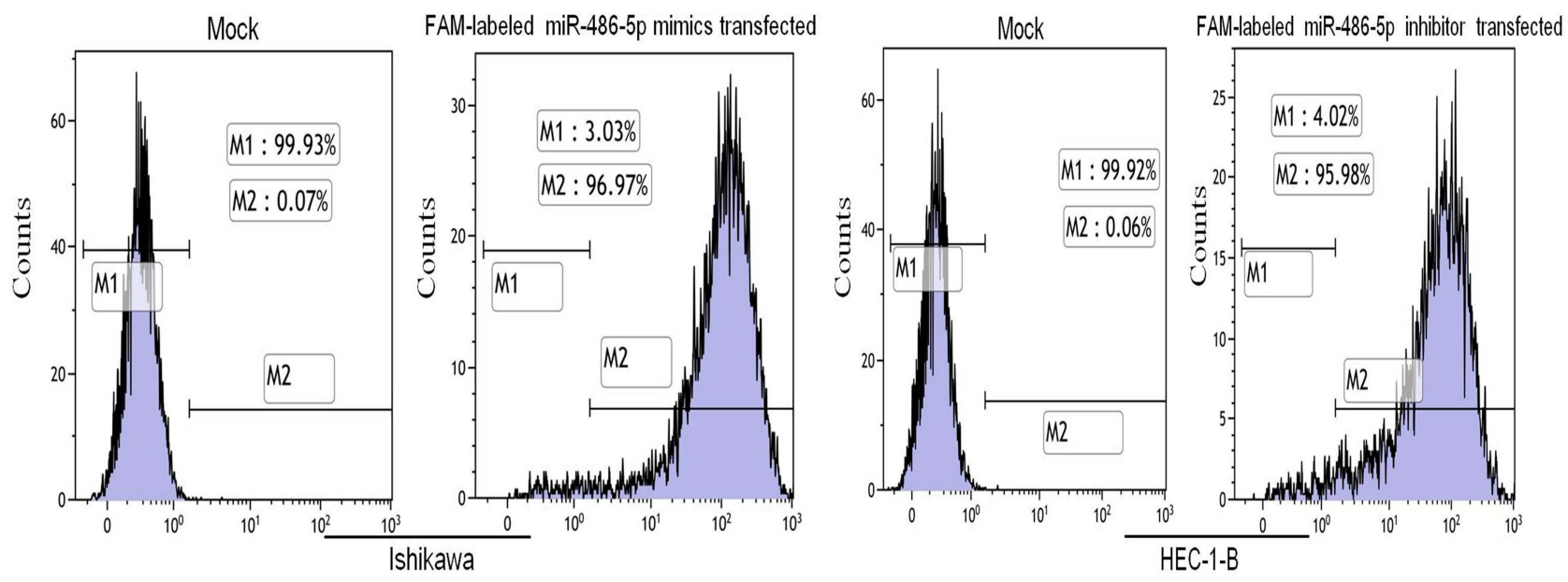

Figure 2 Expression levels of miR-486-5p in cell lines and the efficiency of transfected cells. (A) Relative expression of miR-486-5p in EC cell lines, analyzed by RT-qPCR. $* * * \mathrm{P}<0.00 \mathrm{I}$. (B) Cells were harvested $24 \mathrm{~h}$ after transfection and analyzed on a flow cytometer. (C) The level of miR-486-5p was determined by RT-PCR, the fold-change of miR-486-5p level was determined by the $2^{-\Delta \Delta C t}$ method. Cell lines were transfected with miR-486-5p mimic or inhibit, and their corresponding NC. Data are expressed as the mean \pm SD. $* * P<0.05$, $* * * P<0.001$.

To investigate the possibility that the upregulated expression of miR-486-5p in Ishikawa cells promotes migration and invasion, while its downregulated expression in HEC-1-B cells suppressed their migration and invasion. Figure 5(A-D), wound-healing assay. Representative images show in Figure 5(A and B). As shown in Figure 5(A and $\mathrm{C}$ ), the scratched width of Ishikawa cells transfected with miR-486-5p mimics was remarkably reduced after $24 \mathrm{~h}$ and $48 \mathrm{~h}$ of transfection when compared with negative control group, and so the migratory ability was remarkably promoted $(* * \mathrm{P}<0.01)$. As shown in Figure 5(B and D), the scratched width of HEC-1-B cells transfected with miR-486-5p inhibitors was significantly wider after $24 \mathrm{~h}$ and $48 \mathrm{~h}$ when compared with negative control group, and so the migratory ability was shown to be significantly inhibited $(* * \mathrm{P}<0.01$, $* * * \mathrm{P}<0.001)$; Figure $5(\mathrm{E}-\mathrm{H})$, through matrigel invasive assay. As shown in Figure 5(E and G), the representative images showed that the cell invasion of Ishikawa cells transfected with miR-486-5p mimics was significantly increased when compared with negative control group, and miR-486-5p expression upregulation promoted the invasiveness of Ishikawa cells $\left({ }^{* *} \mathrm{P}<0.01\right)$. As shown in Figure 5(F and $\mathrm{H})$, decreased cell invasiveness of HEC1-B cells transfected with miR-486-5p inhibitors was observed when compared with negative control group, and so downregulated miR-486-5p expression inhibited HEC-1-B cell invasiveness $(* * * \mathrm{P}<0.001)$.

To investigate the molecular mechanisms related to miR486-5p functions in EC, its potential targets were predicted by bioinformatics programs[miRanda (http://www.miRanda. org), TargetScan (http://www.targetscan.org) and miRWalk (http://www.miRWalk.org)], and selected showed association with the phenotype of miR-486-5p. MARK1 was shown to be the target gene whether regulated by miR-486-5p in EC for target validation (Figure 6). Figure 6(A) presents a layout of luciferase reporter constructs, ie, double-stranded oligonucleotides were bound to a putative miR-486-5p site within 
A

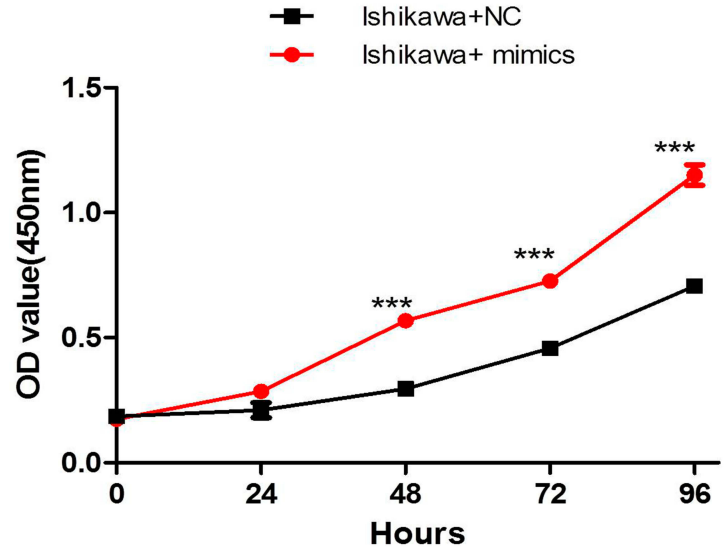

C

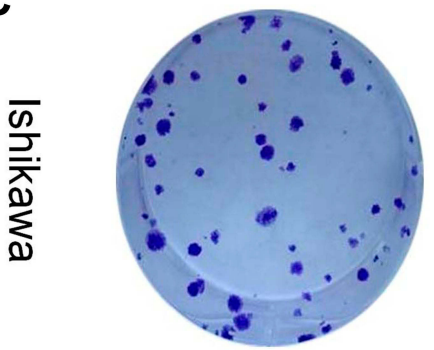

Mock

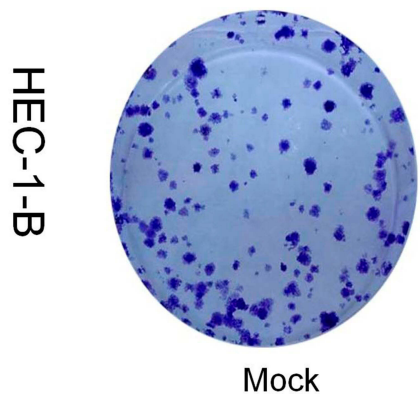

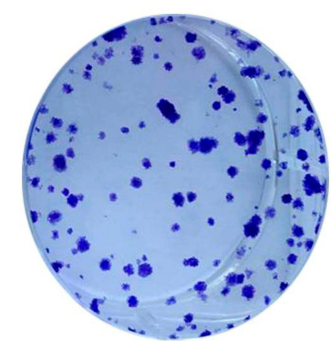

miR-486-5p mimics transfected

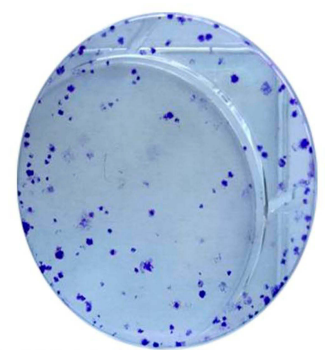

miR-486-5p inhibitor transfected
B

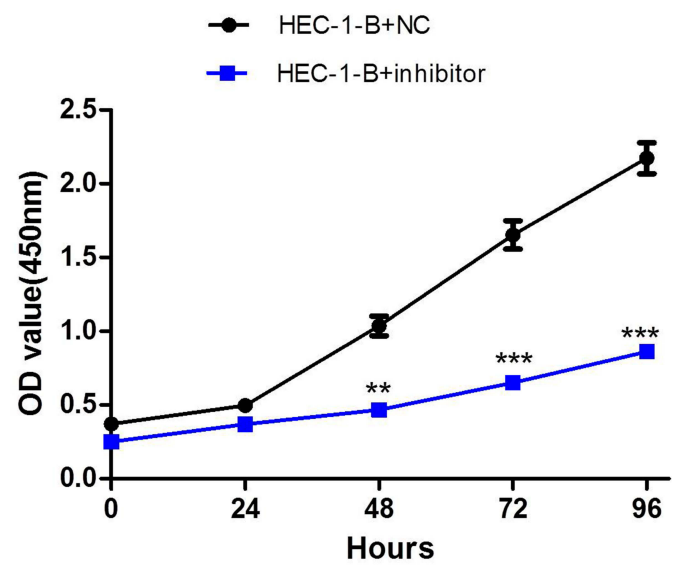

D
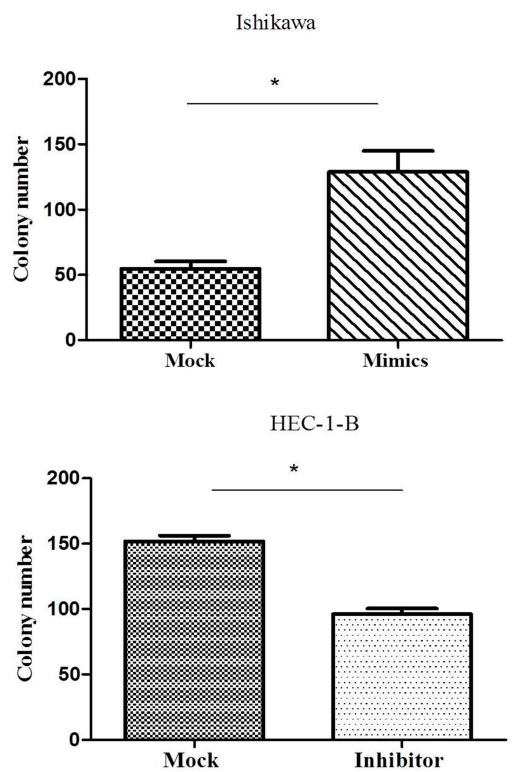

Figure 3 MiR-486-5p promoted proliferation of EC cells. (A and B) CCK-8 assay of Cell viability, relative cell proliferation at $0,24,48$ and $96 \mathrm{~h}$ post-transfection. ${ }^{\text {*** }} \mathrm{P}<0.0 \mathrm{I}$, ${ }^{* * *} \mathrm{P}<0.00 \mathrm{I}$. (C and $\left.\mathbf{D}\right)$ Cell viability was detected by colony formation assay. Data are expressed as the mean $\pm \mathrm{SD}$. ${ }^{*} \mathrm{P}<0.05$.

MARK1 mRNA 3'-UTR. To explore whether miR-486-5p directly targets MARK1, luciferase reporter assays were performed to examine the direct binding sites of miR-486-5p in its 3'UTR, Figure 6(B). The luciferase activity of MARK1_wt with miR-486-5p mimic showed significant reduction $(\sim 50 \%)$ (**P $<0.01)$, indicating that miR-486-5p targets MARK1 through miR-486-5p binding sites within the MARK1 3'UTR. To experimentally explore whether bona fide targeting of MARK1 by miR-486-5p results from miRNA-induced mRNA degradation, the Ishikawa cells were transfected with miR-486-5p mimics and HEC-1-B cells with miR-486-5p inhibitors, Figure 6(C). qPCR analysis was performed to evaluate MARK1 mRNA levels of transfected cells. The results revealed that transfection with miR-486-5p mimics has significantly modified MARK1 downtranscript levels in
Ishikawa cells ( $* * \mathrm{P}<0.01$ ), and miR-486-5p inhibitors have significantly modified MARK1 uptranscript levels in HEC$1-\mathrm{B}$ cells $\left({ }^{* * \mathrm{P}}<0.01\right)$, suggesting the involvement of a biotranslational process within mRNA expression. Figure 6 (D). Ishikawa cells transfected with miR-486-5p mimics and HEC-1-B cells transfected with miR-486-5p inhibitors were assayed $48 \mathrm{~h}$ after transfection by Western blotting, the level of $\beta$-actin served as a loading control, MARK1 protein expression was shown to be changed.

\section{Discussion}

The present study demonstrated that miRNAs are considered as diagnostic and prognostic biomarkers in EC owing to their entanglement in neoplastic initiation and progression. ${ }^{21}$ An increasing trend of miR-486-5p was 

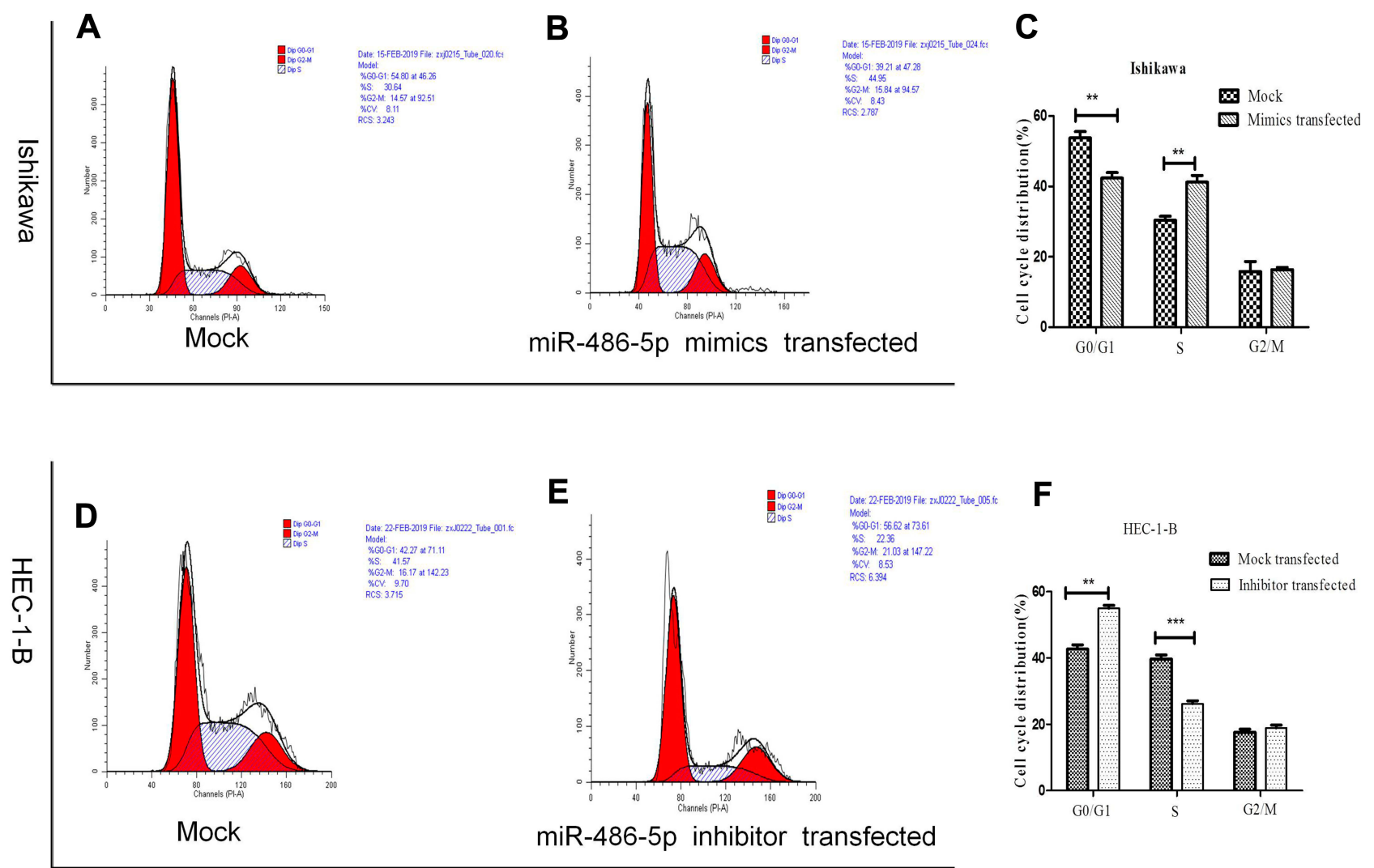

Figure 4 MiR-486-5p changes GI to S phase transition of cell cycle of EC cells. (A-F) Cell cycle changes detected by flow cytometry. Data are expressed as the mean \pm SD. ${ }^{* * *} \mathrm{P}<0.01,{ }^{* * * *} \mathrm{P}<0.001$.

discovered in several types of cancers, and is considered to be involved in various bio-effects such as transcriptional and epigenetic regulations. We herein showed that the (i) expression of miR-486-5p in both serum and tumor tissue was elevated in EC patients; (ii) miR-486-5p is a putative binder of MARK1-3' UTR; (iii) manipulation of miR-486$5 p$ levels can affect the expression levels of MARK1; and (iv) suppression of miR-486-5p expression has significantly attenuated proliferation, colony formation, migration, and invasion of an EC cell line (HEC-1-B) in vitro. Overexpression of miR-486-5p in another EC cell line (Ishikawa) stimulated cell proliferation, colony formation, migration, and invasion in vitro. Collectively, there is no clear conclusion on the association of miR-486-5p and EC to the best of our knowledge. Our results for the first time showed that miR-486-5p regulated tumor progression by regulating the function of MARK1 in EC.

Currently, there are several up or down-regulated miRNAs in EC that are considered to influence cancer initiation, pathogenesis and progression, ${ }^{21}$ and so miRNAs are being considered as new candidate therapeutic targets. The relationship between miRNAs and carcinogenesis is a key aspect in demonstrating the epigenetic effects. ${ }^{22}$ Tumor proliferation and metastases are the leading causes of death in EC. However, the molecular mechanism that underlies the miRNAs still remains inconclusive. So far, the miRNAs have simultaneously functioned in the modification of multiple targets, and miR-486-5p can specifically target various genes in the involvement of tumor progressions such as fibrillin-1 in papillary thyroid carcinoma, ${ }^{23}$ PTEN in cervical cancer, ${ }^{16}$ OLFM4 in gastric cancer ${ }^{14}$ and PIK3R1 in nonsmall cell lung cancer. ${ }^{24}$ Our previous study has remarkably demonstrated that overexpression of miR-486-5p promoted proliferation, migration and invasion in cervical cancer. ${ }^{16}$ Furthermore, miR-486-5p acts as an oncogene in chronic myeloid leukemia progenitors and up-regulation of miR-486-5p expression has significantly promoted the growth, survival and drug sensitivity. However, overexpression of miR-486-5p has effectively inhibited cell proliferation and invasion and successfully induced apoptosis in vitro in non-small cell lung cancer. ${ }^{24}$ Therefore, miR-486-5p function in cells promotes or inhibits proliferation and showed association with a wide spectrum of 

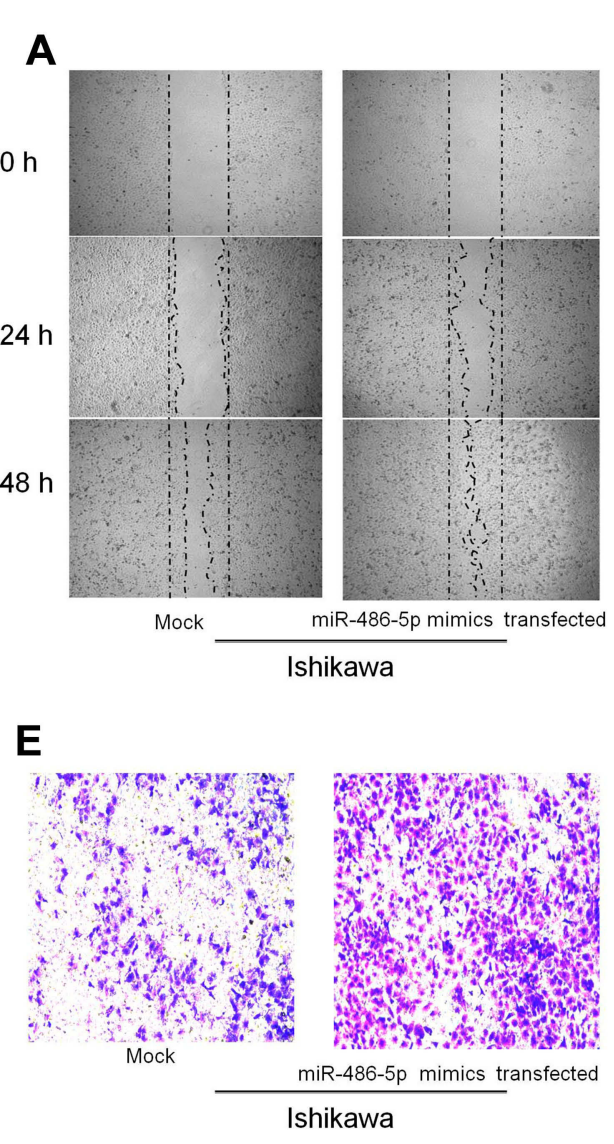

B

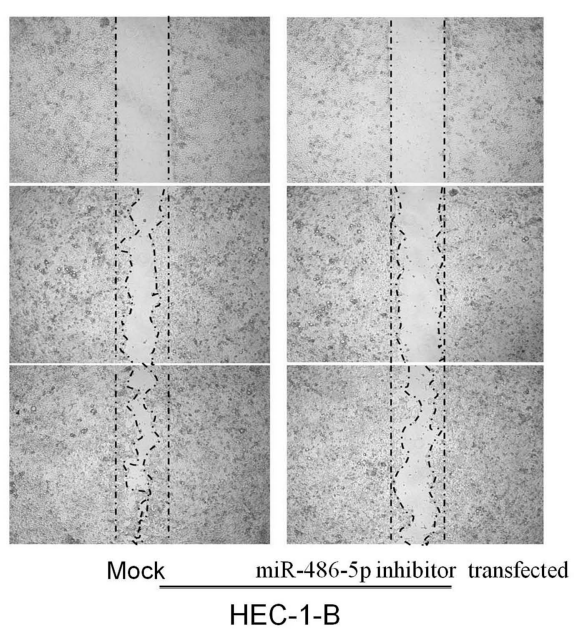

$F$

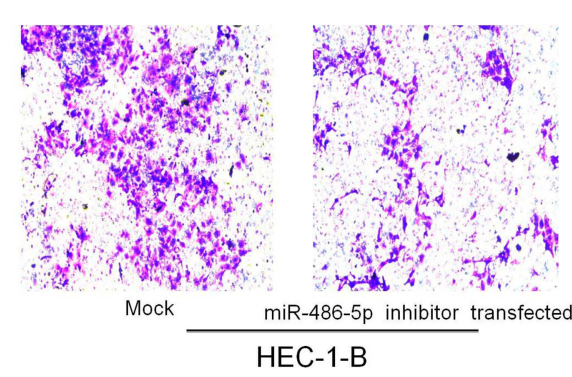

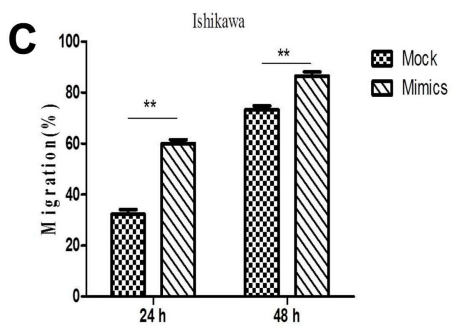

D

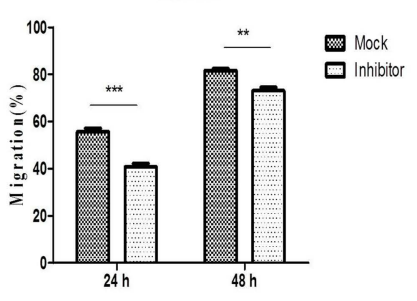

G

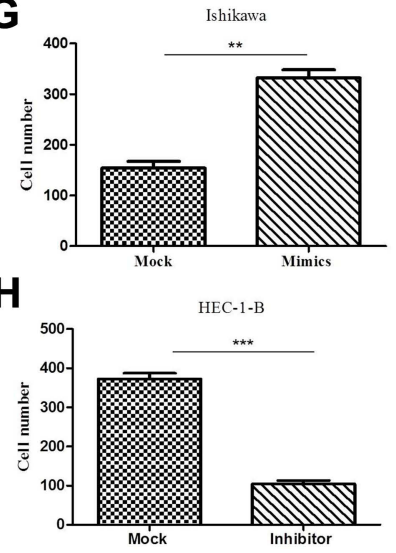

Figure 5 MiR-486-5p promoted migration and invasion of EC cells. (A-D) Representative images of wound-healing assay. ${ }^{* * *} \mathrm{P}<0.0 \mathrm{I},{ }^{* * * *} \mathrm{P}<0.00 \mathrm{I}$. (E-H) Representative images showing matrigel invasive assay. Data are expressed as the mean $\pm S D$. ${ }^{* * *} \mathrm{P}<0.01,{ }^{* * * *} \mathrm{P}<0.001$.

targets. This paper provides results that miR-486-5p-MARK1 signaling pathway is the functional mechanism by which miR486-5p promotes EC.

There are four MARK isoforms (MARK1-4), and they play several roles in regulating cell polarization and microtubule dynamics, which in turn are associated with cell movement in cancer and its development, promotion of cell migration and invasiveness of carcinoma cells. ${ }^{25}$ Among the multiple molecular mechanisms that are altered in human cancers, those that are involved in cell division cycle control are believed to be the main for the process of oncogenesis. ${ }^{26}$ Our results showed the complex bio-effects of MARK1 on cell growth, migration and invasive pathways mediated by miR-486-5p, which were finally connected to each other. Like other protein kinases, MARKs play a role in various signaling pathways and both are regulated by multiple mechanisms, and also through MAP phosphorylation or non-canonical pathways, promoting Wnt activation ${ }^{27}$ and LKB1 signaling $^{28}$ that might benefit cancer research.

MARK1 binds to other "activating" proteins or membrane-bound targets. The gain of function of this MAP in epithelial cells is regulated by MARK1 by inducing microtubule dynamics ${ }^{29,30}$ and by binding to the microtubule lattice, which can stabilize/destabilize the microtubules through dephosphorylation/phosphorylation cycles. Recently, MARK1 functions as a tumor suppressor in several types of cancers. microRNA-23a $\mathrm{a}^{31}$ and miR$125 \mathrm{a}-5 \mathrm{p}^{25}$ promotes cancer cell migration and proliferation by targeting at MARK1, and our study revealed that miR486-5p acts as an oncogene in the progression of EC by influencing MARK1.

In the present study, the miR-486-5p expression levels were markedly varied between EC and normal tissues. A larger sample size to further verify the function in EC is warranted in the future. Collectively, our results for the 


\section{A}

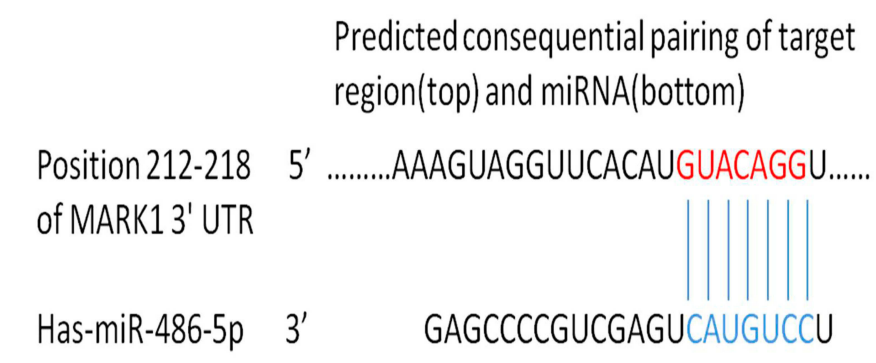

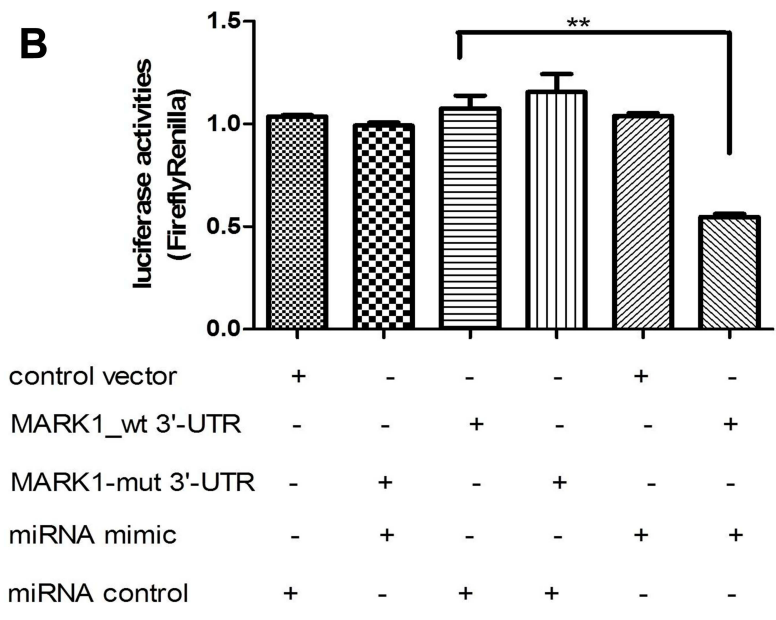

C

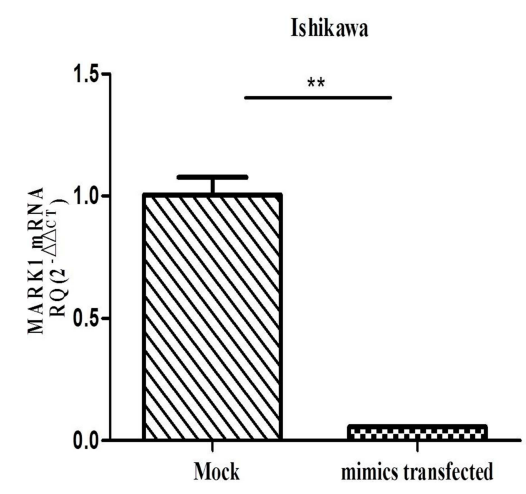

D Mock inhibitor

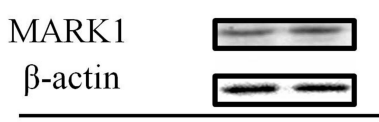

HEC-1-B

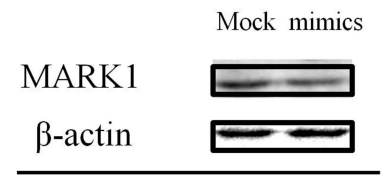

Ishikawa

Figure 6 Identification of miR-486-5p mRNA targets. (A) Sequence alignment of miR-486-5p and its conserved target site in the MARKI 3'-UTR. (B) Luciferase activity, measured in Ishikawa cells with a dual-luciferase reporter assay. ${ }^{*} P<0.01$. (C) qRT-PCR analysis of mRNA expression of MARKI in miR-486-5p (mimic or inhibited) with their corresponding NC, gapdh served as a loading control. ${ }^{* *} \mathrm{P}<0.0 \mathrm{I}$. (D) Western blot of MARKI expression in miR-486-5p (mimic or inhibited), with corresponding NC, b-actin served as control. Data are expressed as the mean \pm SD.

first time showcased that miR-486-5p regulated tumor progression through MARK1 function regulation. There are many factors that are related to the regulation of functional dynamics of EC, and whether any other signaling pathways played a role via miR-486-5p in EC was not determined. MARK1 induced the regulation of G1/S transition and the invasion system. Additional analyses are required to precisely define on how miR-486-5p induced MARK1 activation and how MARK1 regulated signaling of cell proliferation, and invasion. To address these questions and to further understand the miR-486-5p-mediated MARK1 signaling, further research should be conducted.

\section{Conclusions}

In summary, miR-486-5p functions as potential tumor stimulative factors for endometrial carcinoma progression. And MARK1 was identified as a specific target for miR486-5p playing important roles in malignant transformation.
MiR-486-5p expression inversely correlated with MARK1 expression, leads to downregulation of MARK1 consequently influencing cell proliferation, migration and invasion, suggesting a potential role for diagnosis and treatment in endometrial carcinoma tumorigenesis.

\section{Acknowledgments}

Project supported by the Ningbo Health Branding Subject Fund (PPXK2018-04), the Medical and Health Plan of Zhengjiang (grant number 2019KY166), and the Natural Science Foundation of Ning Bo (2019A610306). We would like to thank the Central Laboratory of Ningbo First Hospital.

\section{Disclosure}

Xiaojiao Zheng and Kejun $\mathrm{Xu}$ are co-first authors. The authors report no conflicts of interest in this work. 


\section{References}

1. Siegel RL, Miller KD, Jemal A. Cancer statistics, 2017. CA Cancer J Clin. 2017;67(1):7-30. doi:10.3322/caac.21387

2. Bokhman JV. Two pathogenetic types of endometrial carcinoma. Gynecol Oncol. 1983;15(1):10-17. doi:10.1016/0090-8258(83)90111-7

3. Bansal N, Yendluri V, Wenham RM. The molecular biology of endometrial cancers and the implications for pathogenesis, classification, and targeted therapies. Cancer Control. 2009;16(1):8-13. doi: $10.1177 / 107327480901600102$

4. Bartel DP. MicroRNAs: target recognition and regulatory functions. Cell. 2009;136(2):215-233. doi:10.1016/j.cell.2009.01.002

5. Pasquinelli AE. MicroRNAs and their targets: recognition, regulation and an emerging reciprocal relationship. Nat Rev Genet. 2012;13 (4):271-282. doi:10.1038/nrg3162

6. Hwang HW, Mendell JT. MicroRNAs in cell proliferation, cell death, and tumorigenesis. Br J Cancer. 2006;94(6):776-780. doi:10.1038/sj. bjc. 6603023

7. Calin GA, Croce CM. MicroRNA signatures in human cancers. Nat Rev Cancer. 2006;6(11):857-866. doi:10.1038/nrc1997

8. Perge P, Nagy Z, Igaz I, Igaz P. Suggested roles for microRNA in tumors. Biomol Concepts. 2015;6(2):149-155. doi:10.1515/bmc2015-0002

9. Kanekura K, Nishi H, Isaka K, Kuroda M. MicroRNA and gynecologic cancers. J Obstet Gynaecol Res. 2016;42(6):612-617. doi:10.1111/jog. 12995

10. Wang J, Tian X, Han R, et al. Downregulation of miR-486-5p contributes to tumor progression and metastasis by targeting protumorigenic ARHGAP5 in lung cancer. Oncogene. 2014;33(9):1181-1189. doi:10.1038/onc.2013.42

11. Yi Y, Lu X, Chen J, et al. Downregulated miR-486-5p acts as a tumor suppressor in esophageal squamous cell carcinoma. Exp Ther Med. 2016;12(5):3411-3416. doi:10.3892/etm.2016.3783

12. Tahiri A, Leivonen SK, Luders $\mathrm{T}$, et al. Deregulation of cancer-related miRNAs is a common event in both benign and malignant human breast tumors. Carcinogenesis. 2014;35(1):76-85. doi:10.1093/carcin/bgt333

13. Li H, Mou Q, Li P, et al. MiR-486-5p inhibits IL-22-induced epithelial-mesenchymal transition of breast cancer cell by repressing Dock1. J Cancer. 2019;10(19):4695-4706. doi:10.7150/jca.30596

14. Oh HK, Tan AL, Das K, et al. Genomic loss of miR-486 regulates tumor progression and the OLFM4 antiapoptotic factor in gastric cancer. Clin Cancer Res. 2011;17(9):2657-2667. doi:10.1158/10780432.CCR-10-3152

15. Liu X, Chen X, Zeng K, et al. DNA-methylation-mediated silencing of miR-486-5p promotes colorectal cancer proliferation and migration through activation of PLAGL2/IGF2/beta-catenin signal pathways. Cell Death Dis. 2018;9(10):1037. doi:10.1038/s41419018-1105-9

16. Li C, Zheng X, Li W, Bai F, Lyu J, Meng QH. Serum miR-486-5p as a diagnostic marker in cervical cancer: with investigation of potential mechanisms. BMC Cancer. 2018;18(1):61. doi:10.1186/s12885-017$3753-\mathrm{z}$
17. Goto K, Oue N, Shinmei S, et al. Expression of miR-486 is a potential prognostic factor after nephrectomy in advanced renal cell carcinoma. Mol Clin Oncol. 2013;1(2):235-240. doi:10.3892/ mco.2012.46

18. He Y, Liu J, Wang Y, et al. Role of miR-486-5p in regulating renal cell carcinoma cell proliferation and apoptosis via TGF-betaactivated kinase 1. J Cell Biochem. 2019;120(3):2954-2963. doi: $10.1002 /$ jcb. 26900

19. Wang LS, Li L, Li L, et al. MicroRNA-486 regulates normal erythropoiesis and enhances growth and modulates drug response in CML progenitors. Blood. 2015;125(8):1302-1313. doi:10.1182/ blood-2014-06-581926

20. Sclafani RA, Holzen TM. Cell cycle regulation of DNA replication. Аnnu Rev Genet. 2007;41:237-280. doi:10.1146/annurev. genet.41.110306.130308

21. Umene K, Banno K, Kisu I, et al. New candidate therapeutic agents for endometrial cancer: potential for clinical practice (review). Oncol Rep. 2013;29(3):855-860. doi:10.3892/or.2013.2221

22. Toh TB, Lim JJ, Chow EK. Epigenetics in cancer stem cells. Mol Cancer. 2017;16(1):29.

23. Ma X, Wei J, Zhang L, et al. miR-486-5p inhibits cell growth of papillary thyroid carcinoma by targeting fibrillin-1. Biomed Pharmacother. 2016;80:220-226. doi:10.1016/j.biopha.2016.03.020

24. Tian F, Wang J, Ouyang T, et al. MiR-486-5p serves as a good biomarker in nonsmall cell lung cancer and suppresses cell growth with the involvement of a target PIK3R1. Front Genet. 2019;10:688. doi:10.3389/fgene. 2019.00688

25. Natalia MA, Alejandro GT, Virginia TJ, Alvarez-Salas LM. MARK1 is a novel target for miR-125a-5p: implications for cell migration in cervical tumor cells. Microrna. 2018;7(1):54-61. doi:10.2174/ 2211536606666171024160244

26. Manning AL, Dyson NJ. pRB, a tumor suppressor with a stabilizing presence. Trends Cell Biol. 2011;21(8):433-441. doi:10.1016/j. tcb.2011.05.003

27. Wallingford JB, Habas R. The developmental biology of Dishevelled: an enigmatic protein governing cell fate and cell polarity. Development. 2005;132(20):4421-4436. doi:10.1242/dev.02068

28. Goodwin JM, Svensson RU, Lou HJ, Winslow MM, Turk BE, Shaw RJ. An AMPK-independent signaling pathway downstream of the LKB1 tumor suppressor controls Snaill and metastatic potential. Mol Cell. 2014;55(3):436-450. doi:10.1016/j.molcel.2014.06.021

29. Hammond JW, Huang CF, Kaech S, Jacobson C, Banker G, Verhey KJ. Posttranslational modifications of tubulin and the polarized transport of kinesin-1 in neurons. Mol Biol Cell. 2010;21 (4):572-583. doi:10.1091/mbc.e09-01-0044

30. Witte H, Bradke F. The role of the cytoskeleton during neuronal polarization. Curr Opin Neurobiol. 2008;18(5):479-487. doi:10.1016/j.conb.2008.09.019

31. Tang X, Yang M, Wang Z, Wu X, Wang D. MicroRNA-23a promotes colorectal cancer cell migration and proliferation by targeting at MARK1. Acta Biochim Biophys Sin (Shanghai). 2019;51 (7):661-668. doi:10.1093/abbs/gmz047

\section{Publish your work in this journal}

OncoTargets and Therapy is an international, peer-reviewed, open access journal focusing on the pathological basis of all cancers, potential targets for therapy and treatment protocols employed to improve the management of cancer patients. The journal also focuses on the impact of management programs and new therapeutic agents and protocols on patient perspectives such as quality of life, adherence and satisfaction. The manuscript management system is completely online and includes a very quick and fair peer-review system, which is all easy to use. Visit http://www.dovepress.com/ testimonials.php to read real quotes from published authors. 